\title{
Guided and unguided self-help for social anxiety disorder: randomised controlled trial
}

Tomas Furmark, Per Carlbring, Erik Hedman, Annika Sonnenstein, Peder Clevberger,

Benjamin Bohman, Anneli Eriksson, Agneta Hållén, Mandus Frykman, Annelie Holmström,

Elisabeth Sparthan, Maria Tillfors, Elisabeth Nilsson Ihrfelt, Maria Spak, Anna Eriksson,

Lisa Ekselius and Gerhard Andersson

\section{Background}

Internet-delivered self-help programmes with added therapist guidance have shown efficacy in social anxiety disorder, but unguided self-help has been insufficiently studied.

\section{Aims}

To evaluate the efficacy of guided and unguided self-help for social anxiety disorder.

\section{Method}

Participants followed a cognitive-behavioural self-help programme in the form of either pure bibliotherapy or an internet-based treatment with therapist guidance and online group discussions. A subsequent trial was conducted to evaluate treatment specificity. Participants $(n=235)$ were randomised to one of three conditions in the first trial, or one of four conditions in the second.

\section{Results}

Pure bibliotherapy and the internet-based treatment were better than waiting list on measures of social anxiety, general anxiety, depression and quality of life. The internetbased therapy had the highest effect sizes, but directly comparable effects were noted for bibliotherapy augmented with online group discussions. Gains were well maintained a year later.

\section{Conclusions}

Unguided self-help through bibliotherapy can produce enduring improvement for individuals with social anxiety disorder.

\section{Declaration of interest}

T.F., A.H., E.S., P.Ca. and G.A. receive royalties for authoring the self-help book used for bibliotherapy.
Internet-delivered cognitive-behavioural therapy (CBT) has developed rapidly, and emerging evidence suggests that text-based self-help manuals presented via the internet and supported by therapist feedback can yield reliable improvements in a variety of mental disorders. ${ }^{1,2}$ Apart from reducing costs for office locations, travel and staff, internet-based treatments may be of great value for people living in remote areas, for those with irregular or inconvenient work schedules, and for individuals who fear or cannot afford face-to-face therapy. In Sweden we have previously developed and tested the effects of internet-delivered treatment for social anxiety disorder (social phobia); results from one open trial and three randomised controlled trials indicate short-term and long-term effects that are in line with CBT delivered in the traditional manner. ${ }^{3-8}$ This has been replicated in Australia. ${ }^{9-11}$ A crucial question, however, is how the treatment would work when presented as pure bibliotherapy, i.e. as a self-help book without additional therapist guidance. Two recent studies showed only limited efficacy of pure self-help for social anxiety disorder, ${ }^{11,12}$ although this form of treatment has been successful for other disorders. ${ }^{13}$ There may be other ways to provide support in bibliotherapy, for example by using online discussion groups. The main objective of our study was to evaluate the efficacy of pure bibliotherapy compared with a multimodal, internet-delivered cognitive-behavioural therapy (ICBT) package and a waiting-list control condition. To test the robustness and specificity of the treatments, a subsequent trial was performed that included two additional treatment arms: bibliotherapy augmented with an online discussion group and internet-delivered applied relaxation.

\section{Method}

\section{Recruitment and participants}

The general procedure was similar to our previous randomised controlled trials of internet-delivered self-help for social anxiety disorder. ${ }^{4-6}$ Participants were recruited by media advertisements and internet links from the Swedish National Anxiety Association and the Swedish Association for Behaviour Therapy during the spring and autumn of 2005. A web page was created which included an outline of the study as well as general information about social anxiety disorder, CBT, ethical issues, internet security and a description of the study personnel. Participants filled out an application form and a computerised screening battery consisting of the Social Phobia Screening Questionnaire (SPSQ), ${ }^{14}$ the selfrated version of the Montgomery \& Åsberg Depression Rating Scale (MADRS-S), ${ }^{15}$ and additional questions regarding current and past treatment. To be included, participants had to meet the following criteria:

(a) a DSM-IV diagnosis of social anxiety disorder, ${ }^{16}$ according to the SPSQ;

(b) scoring below 31 on the MADRS-S depression scale and below 4 on the suicide item of this scale (to prevent the inclusion of individuals in strong need of specialist consultation);

(c) not undergoing any other psychological treatment during the study period;

(d) if prescribed drugs for anxiety or depression, the dosage had to be constant for 3 months before the treatment onset and kept constant throughout the study;

(e) being at least 18 years old;

(f) living in Sweden;

(g) having access to a computer with internet connection;

(h) not admitting another serious or dominant disorder (e.g. psychosis, substance misuse) that could be expected to influence the outcome of the study; 
(i) having a primary diagnosis of social anxiety disorder according to the Structured Clinical Interview for DSM-IV Axis I Disorders (SCID-I). ${ }^{17}$

The last criterion was evaluated by a telephone interview in which the diagnostic questions from the social anxiety disorder section of the SCID-I were posed. When a person failed to meet the inclusion criteria, an individual letter was sent with advice on where to seek more appropriate help.

Of the 580 individuals who applied to participate, 235 individuals meeting all inclusion criteria were eventually randomised to one of three arms in trial 1 (ICBT, bibliotherapy alone or waiting list) or one of four arms (ICBT, bibliotherapy alone, bibliotherapy with discussion group or internet-delivered applied relaxation) in trial 2 (Fig. 1). Randomisation was performed by an independent third party using an online true random-number service (www.random.org). Two participants, one each from the pure bibliotherapy and waiting-list groups, withdrew immediately after randomisation because of personal reasons and one additional participant (ICBT group) did not provide post-treatment data. The waiting-list control group received delayed treatment after 9 weeks (see below) but 3 individuals withdrew shortly before this treatment began. Oneyear follow-up data were not provided by 34 participants (14.5\%). For all randomised participants, missing data were replaced by the last obtained score (pre- or post-treatment), i.e. last observation carried forward.

Ten participants (4.3\%) withdrew from the study after the first $(n=6)$ or second $(n=4)$ treatment week, the main reasons being lack of time or motivation and personal problems unrelated to the treatment. In accordance with the intention-to-treat principle, all participants were asked to complete post-treatment and follow-up assessments, regardless of how many treatment modules they had completed.

The trials were approved by the regional ethics committee and written informed consent was obtained from all participants.

\section{Outcome measures}

Four social anxiety questionnaires were used as primary outcome measures: the Liebowitz Social Anxiety Scale self-report version (LSAS-SR), ${ }^{18}$ the Social Phobia Scale (SPS), ${ }^{19}$ the Social Interaction Anxiety Scale (SIAS) ${ }^{19}$ and the SPSQ. In addition, three secondary measures were used to measure general anxiety, depression and quality of life: the Beck Anxiety Inventory (BAI) ${ }^{20}$ the MADRS-S and the Quality of Life Inventory (QoLI). ${ }^{21}$ All instruments were administered via the internet before treatment (baseline), immediately after treatment and 1 year later. Adequate psychometric properties have previously been demonstrated for internet-administered questionnaires. ${ }^{22}$ In addition, all participants completed the LSAS-SR online every week (on Sundays) in order to monitor weekly treatment gains. In cases of missing data, a brief, neutral reminder was sent $24 \mathrm{~h}$ later by email, and if necessary followed by another reminder sent as an autogenerated short text message (SMS) to the person's mobile telephone (cellphone). The response rate on these weekly assessments ranged between $80 \%$ and $100 \%$ across modules, and the last available LSAS-SR rating was used to replace missing data.

\section{Treatment credibility ratings}

After the first week of treatment, i.e. after the rationale had been presented briefly in the manual, participants answered five

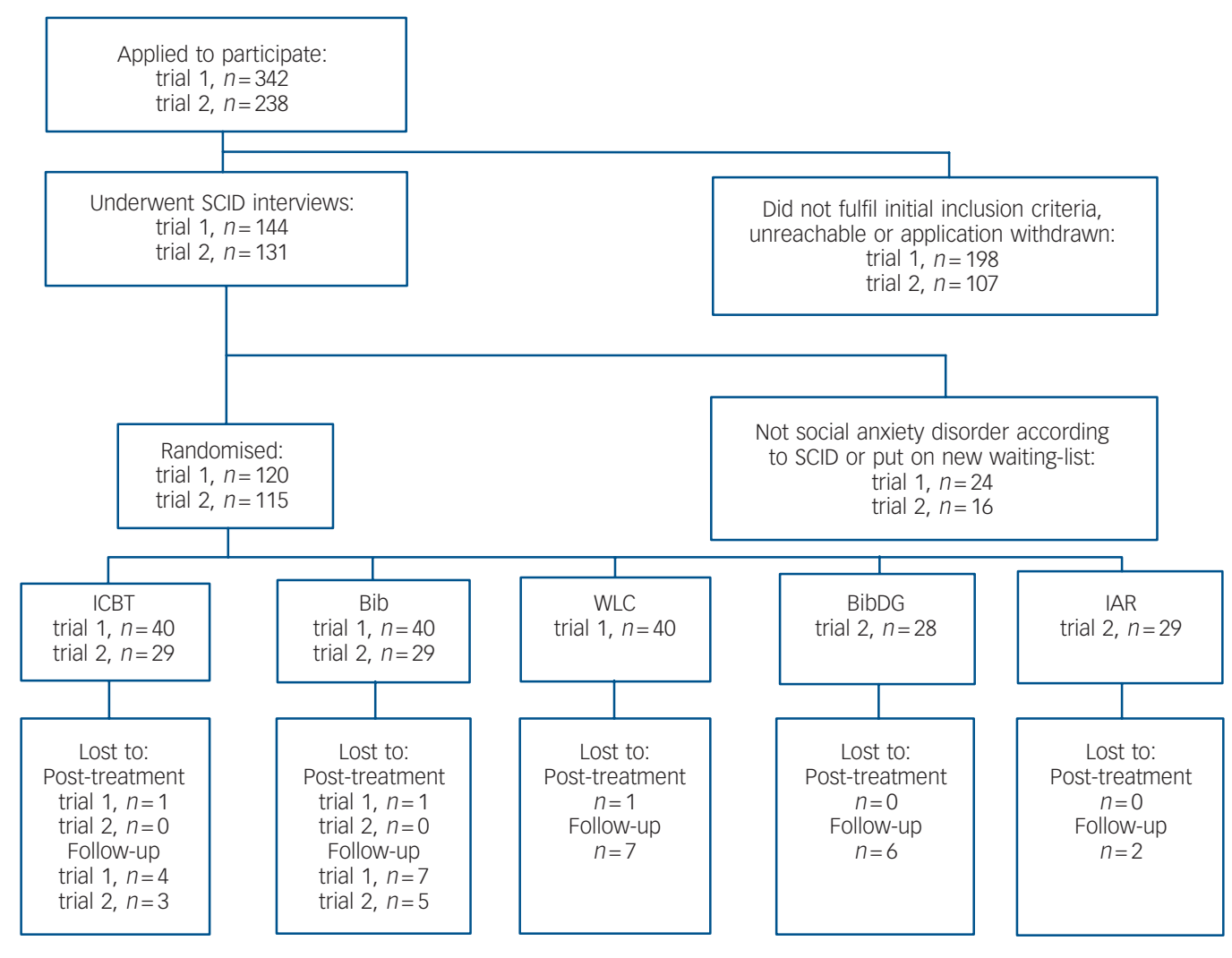

Fig. 1 Participant flow, randomisation and withdrawals at each stage of the study. Bib, pure bibliotherapy; BibDG, bibliotherapy with access to an online discussion group; IAR, internet-delivered applied relaxation; ICBT, internet-delivered cognitive-behavioural therapy; SCID, Structured Clinical Interview for DSM-IV Axis I Disorders; WLC, waiting-list control group treated after 9 weeks (post-treatment). 
questions online regarding their perceptions of the credibility of the treatment to which they had been randomised, for example their overall belief in the treatment method and expectancies of improvement. ${ }^{23}$ The total scores ranged between 5 and 50 .

\section{General treatment procedure}

All participants were required to have access to a computer with an internet connection, an email program and the ability to print out files in pdf format. Participants were advised to use a free online email service that automatically encrypts messages in 2048 bits.

\section{Self-help manual}

The main treatment component was our previously evaluated selfhelp manual for social anxiety disorder, which consisted of 186 pages divided into nine chapters (modules) adapted for use over the internet. ${ }^{3-6}$ The introductory module described social anxiety disorder and facts about CBT. Modules 2-4 described a cognitive model for social anxiety disorder and introduced cognitive restructuring. Modules 5-7 introduced exposure exercises and attention training. Modules 8 and 9 mainly concerned social skills and relapse prevention. The manual was released as a self-help book for the Swedish market after completion of the study. ${ }^{24}$

Participants were asked to complete one module every week, i.e. a 9-week treatment period was required. Each module consisted of information and exercises (homework assignments) and ended with a short quiz to check adherence. Participants were also asked to summarise, in their own words, a central section of the module in question and to describe the outcome of the exercises in a treatment diary (bibliotherapy groups) or in weekly email correspondence with their internet therapist (ICBT groups). The applied relaxation group used another manual but with a similar structure (see below).

\section{Discussion group}

Participants in all groups except the pure bibliotherapy and waiting-list groups had access to an online discussion forum. For each module, participants were asked to post at least one message about a topic related to the module and to share their weekly achievements with the rest of the group. They were also encouraged to provide feedback and support for others. Separate discussion groups were used for each treatment arm. Discussions were surveyed but the study personnel did not take part in them. The discussion group was open during the whole study period from the first day of treatment until the 1-year follow-up assessment, although the activity was very low after the initial 9 weeks.

\section{Internet therapists}

The ICBT and IAR groups had access to an internet therapist during the 9-week treatment period. Email correspondence occurred weekly (Sundays) and generally concerned the results of homework assignments as described in the self-help manual. The rationale behind the homework assignments was to promote learning and enable the internet therapists to decide whether the participants had assimilated the information and completed their exercises. In general, therapist feedback on the homework assignment was given within $24 \mathrm{~h}$ after the participants had sent their answers by email. When the homework was completed, the next module was made accessible. Alternatively, instruction on what needed to be completed to proceed to the next module was sent to the participant. On average, internet therapists spent approximately $15 \mathrm{~min}$ per week giving email feedback to each participant. There were 13 internet therapists in the study, of whom 6 were licensed clinical psychologists and 7 were clinical psychology students in their last semester of the 5-year master's degree programme. The students had clinical supervision during the trial. Participants were randomly allocated to their therapist.

\section{Treatment arms}

Internet-delivered cognitive-behavioural treatment

The ICBT package consisted of our standard internet treatment for social anxiety disorder. ${ }^{3-6}$ Main components were the self-help manual, weekly email feedback from an internet therapist and an online discussion forum. Additional group exposure sessions, as evaluated in previous trials, ${ }^{4,6}$ were not used. Modules in the self-help manual, including practice sheets and weekly interactive multiple choice quizzes, were presented on the web. Modules were also sent by post on a weekly basis to the participants.

\section{Pure bibliotherapy}

Participants in the 'pure' bibliotherapy (Bib) arm received the complete self-help manual for social anxiety disorder by mail, ${ }^{23}$ together with an explanatory letter with instructions to complete one module per week and to fill out the LSAS-SR form online. The manual was thus the same as the one used for ICBT with only minimal changes to the text regarding homework assignments. For example, participants summarised the outcome of the homework assignments in a treatment diary and not in emails to a therapist. Participants had no contact with the study team except for the usual online assessments before, immediately after and 1 year after treatment. As in all groups, some participants received neutral reminders, sent by email or SMS, to complete the LSAS-SR.

\section{Waiting-list control}

The waiting-list control (WLC) group controlled for time and repeated assessments during the initial 9-week period. Participants had no contact with each other or with the study team during their waiting period except for conditional reminders by email or SMS to complete the weekly assessment of the LSAS-SR. Directly after the waiting period, they were treated using the same internet-delivered self-help manual as for the ICBT group. However, therapist feedback was provided either in small online discussion groups or in individual emails without access to a discussion group. One-year follow-up assessments in the WLC group reflect the long-term effects of this treatment.

\section{Bibliotherapy with access to an online discussion group}

The only difference in the bibliotherapy with access to an online discussion group (BibDG) compared with 'pure' bibliotherapy was accessibility to an online discussion forum (see above).

\section{Internet-delivered applied relaxation}

Participants in the internet-delivered applied relaxation (IAR) group followed an applied relaxation manual, ${ }^{25}$ which had been adapted for self-help use via the internet and evaluated (e.g. for panic disorder). ${ }^{26}$ The manual was slightly modified to suit the population with social anxiety disorder. The structure of the manual was the same as in the ICBT and Bib groups, i.e. the text was divided into nine modules containing information and exercises, and participants completed one module per week. The introductory module included information about social anxiety disorder and anxiety reactions. A more thorough rationale for applied relaxation was given in module 2, i.e. a theoretical 
background was presented describing the importance of noticing and preventing anxiety in its early phase. Modules 3-7 included relaxation exercises (progressive, conditioned, differential and quick relaxation) in which participants gradually learned to relax, with the ultimate goal of achieving a relaxed state within 20-30 s. Modules 8 and 9 included applied relaxation exercises in actual phobic situations (in vivo exposure) and relapse prevention. Each module ended with a quiz and homework assignments to be reported to the internet therapist. As in the ICBT groups, participants had access to an online discussion forum and received weekly feedback from their internet therapist, usually within $24 \mathrm{~h}$. Modules were made accessible one by one on the web, and for convenience were also sent by post to the participants on a weekly basis provided that homework assignments had been completed. A compact disc with relaxation instructions was also sent to the participants.

\section{Statistical analysis}

Differential outcomes were evaluated at post-treatment and follow-up by analysis of covariance (ANCOVA) using pretreatment values as the covariate. Within-group $t$-tests were used to evaluate additional improvement from post-treatment to follow-up. Effect sizes were calculated based on the pooled standard deviation (pre- and post-treatment) and expressed as Cohen's $d .{ }^{27}$ Weekly treatment gains were evaluated using repeated-measurement analysis of variance (ANOVA) and Pearson correlations were calculated to evaluate the association between number of modules completed and level of improvement. Clinically significant improvement was determined in accordance with Jacobson \& Truax criteria, ${ }^{28}$ i.e. by using the Reliable Change Index for each individual and a post-treatment score within two standard deviations of the mean of the normal population. Swedish normative data were used for the SPSQ, SPS and SIAS questionnaires, ${ }^{14,29}$ and American norms for the LSAS-SR. ${ }^{30}$ Chi-squared or exact cellwise tests were used to test distribution differences with regard to clinically significant improvement and demographic and descriptive characteristics. Analyses were done using SPSS version 17.0 for Windows.

\section{Results}

\section{Pre-treatment evaluation}

Groups did not differ significantly with regard to age (trial 1: $F=0.73$, d.f. $=2,117, \quad P=0.48$; trial $2: \quad F=0.91$, d.f. $=3,111$,
$P=0.44)$ or other descriptive characteristics in Table 1 , either in trial 1 or in trial $2\left(\chi^{2}=0.03-4.24\right.$, d.f. $\left.=2-3, P=0.99-0.15\right)$. Also, there was no significant pre-treatment difference between the study groups on primary social anxiety measures (trial 1: $F=0.16-0.85$, d.f. $=2,117, P=0.85-0.43$; trial 2: $F=0.74-2.38$, d.f. $=3,111, P=0.53-0.07$ ) or on secondary measures (trial 1: $F=0.29-0.33$, d.f. $=2,117, P=0.75-0.71 ;$ trial $2: F=1.08-2.55$, d.f. $=3,111, \quad P=0.36-0.06)$. All group differences remained insignificant $(P>0.10)$ when data were pooled across trials.

At baseline, groups scored approximately three standard deviations higher than the Swedish normal population ${ }^{14,29}$ on the SPSQ and approximately four standard deviations higher on the SPS and SIAS measures, indicating that participants had clinically significant symptoms of social anxiety disorder.

\section{Treatment effects in trial 1}

Analyses of covariance for post-treatment change, using baseline values as covariates, revealed significant main effects of the group factor (ICBT/Bib/WLC) on all primary $(F=11.78-18.03$, d.f. $=2,116, \quad P<0.0001)$ and secondary $\quad(F=4.98-11.11$, d.f. $=2,116, P<0.009)$ outcome measures, indicating differential improvement over the treatment period. Bonferroni-corrected pairwise comparisons showed that both the ICBT and the Bib groups were significantly more improved in comparison with waiting-list controls on all social anxiety measures $(P<0.001)$ as well as on the BAI $(P \leqslant 0.015)$, MADRS-S $(P \leqslant 0.001)$ and QoLI $(P \leqslant 0.026)$. The ICBT and Bib groups did not differ significantly on any measure $(P>0.10)$. At follow-up 1 year after all participants had received treatment, the group factor was significant only for the SPS $(F=3.16$, d.f. $=2,116, P<0.05)$ with pairwise comparisons indicating a trend for better improvement in ICBT compared with $\operatorname{Bib}(P=0.065)$. All other pairwise comparisons remained insignificant at this time.

Additional improvement from post-treatment test to followup was noted only in the ICBT group on social anxiety measures $(t=2.58-4.12$, d.f. $=39, P=0.013-0.0002)$. The WLC group did not improve from pre- to post-treatment $(t=0.11-1.08$, d.f. $=39, P=0.91-0.29)$, but significant gains between the posttreatment test and 1-year follow-up, i.e. after delayed treatment, were noted on all primary $(t=3.61-5.57$, d.f. $=39, P<0.001)$ and secondary $(t=3.39-3.49$, d.f. $=39, P<0.002)$ measures.

Within-group effect sizes are given in Table 2 for primary measures and in online Table DS1 for secondary measures. For social anxiety measures, effect sizes in the treated groups were

Table 1 Descriptive characteristics of the sample


$0.65-1.29$ post-treatment and $0.75-1.71$ at follow-up relative to baseline, ICBT being associated with the highest effects. Mean within-group effect sizes across all measures, at post-treatment/ follow-up relative to baseline were 0.82/1.07 (ICBT) and 0.64/ 0.76 (Bib). A mean within-group effect size of 0.61 was noted in the WLC condition at follow-up relative to the post-treatment test, i.e. after delayed treatment. Between-group effect sizes posttreatment, averaged across all measures, were 0.63 for ICBT $v$. WLC and 0.64 for Bib $v$. WLC.

\section{Treatment effects in trial 2}

Differential improvement could not be demonstrated because the ANCOVAs did not reveal significant effects of treatment group (ICBT/Bib/BibDG/IAR) on any post-treatment $(F=0.18-1.36$, d.f. $=3,110, P=0.91-0.26)$ or 1-year follow-up $(F=0.05-1.43$, d.f. $=3,110, P=0.98-0.24)$ measurement, and all planned group comparisons remained insignificant $(P>0.10)$. Within-group evaluations showed significant improvement in all groups from pre- to post-treatment $(P<0.05$ on every measure), but no group showed additional improvement between post-treatment and follow-up $(t=1.49$, d.f. $=27-28, P>0.14)$. Within-group effect sizes for change in primary outcomes were $0.65-1.63$ at posttreatment and $0.65-1.58$ at follow-up relative to baseline, the highest effects being observed in BibDG participants (Table 2). At least moderate effect sizes were noted for changes in general anxiety and depression (online Table DS1), but as in trial 1, effect sizes for changes in QoLI scores were somewhat lower. Mean effect sizes across all measures, at post-treatment/follow-up relative to baseline were as follows: 0.99/0.98 (ICBT), 0.74/0.75 (Bib), 0.99/ 1.06 (BibDG), and 0.77/0.84 (IAR).

\section{Pooled analyses and time course of improvement}

Because the relative efficacy of ICBT $v$. Bib was our principal research question, data from trials 1 and 2 were pooled in order to increase sample sizes (to $n=69$ in each group) and thus the statistical power. Data pooling was also motivated by the fact that neither the ICBT nor the Bib arm showed significant differences across trials with regard to primary, secondary or descriptive measures before treatment.

In ANCOVAs for post-treatment change on social anxiety measures, relative to baseline, the group factor (ICBT/Bib) remained insignificant $(F=0.02-0.64$, d.f. $=1,135, P=0.88-0.43)$, indicating similar levels of short-term improvement with the two types of treatment. The corresponding analyses of change between baseline and 1-year follow-up revealed a significant effect of group on the SPS $(F=4.41$, d.f. $=1,135, P=0.038)$ and LSAS-SR $(F=4.41$, d.f. $=1,135, P=0.038)$, and a marginal effect

Table 2 Descriptive scores and effect sizes for social anxiety measures

\begin{tabular}{|c|c|c|c|c|c|}
\hline \multirow[b]{2}{*}{ Group/measure } & \multirow{2}{*}{$\begin{array}{l}\text { Pre-treatment score } \\
\text { Mean (s.d.) }\end{array}$} & \multirow{2}{*}{$\begin{array}{l}\text { Post-treatment score } \\
\text { Mean (s.d.) }\end{array}$} & \multirow{2}{*}{$\begin{array}{l}\text { Score at 1-year follow-up } \\
\text { Mean (s.d.) }\end{array}$} & \multicolumn{2}{|c|}{ Effect size, $d$} \\
\hline & & & & Pre- to post-treatment & Pre-treatment to follow-up \\
\hline \multicolumn{6}{|l|}{ Trial 1} \\
\hline \multicolumn{6}{|l|}{ ICBT $(n=40)$} \\
\hline SPS & 39.15 (15.35) & $25.60(12.22)$ & $20.13(12.90)$ & 0.98 & 1.34 \\
\hline SIAS & $50.98(14.21)$ & $39.03(14.03)$ & $34.48(15.66)$ & 0.85 & 1.10 \\
\hline SPSQ & $32.18(7.16)$ & $22.10(8.47)$ & $18.03(9.23)$ & 1.29 & 1.71 \\
\hline LSAS-SR & $71.30(22.49)$ & $50.98(21.12)$ & $40.53(20.19)$ & 0.93 & 1.44 \\
\hline \multicolumn{6}{|l|}{ Bib $(n=40)$} \\
\hline SPS & $36.58(15.43)$ & $25.90(16.32)$ & $24.62(17.26)$ & 0.67 & 0.73 \\
\hline SIAS & $48.15(14.61)$ & $38.25(15.84)$ & $36.98(16.34)$ & 0.65 & 0.72 \\
\hline SPSQ & $30.63(7.99)$ & $21.93(11.32)$ & $20.50(10.74)$ & 0.89 & 1.07 \\
\hline LSAS-SR & $68.68(23.87)$ & $48.50(27.46)$ & 45.85 (25.89) & 0.78 & 0.92 \\
\hline \multicolumn{6}{|l|}{ WLC $(n=40)$} \\
\hline SPS & $36.35(17.10)$ & $35.60(16.16)$ & $23.60(16.60)$ & 0.05 & 0.76 \\
\hline SIAS & $46.45(17.90)$ & $46.58(18.45)$ & $34.85(18.48)$ & -0.01 & 0.64 \\
\hline SPSQ & 30.28 (10.33) & 29.73 (11.83) & $22.22(14.63)$ & 0.05 & 0.64 \\
\hline LSAS-SR & 71.28 (24.93) & $70.25(27.25)$ & $47.08(30.20)$ & 0.04 & 0.87 \\
\hline \multicolumn{6}{|l|}{ Trial 2} \\
\hline \multicolumn{6}{|l|}{ ICBT $(n=29)$} \\
\hline SPS & $35.34(17.04)$ & $22.00(16.07)$ & 19.55 (16.79) & 0.81 & 0.93 \\
\hline SIAS & $51.93(14.43)$ & $36.14(16.06)$ & $33.48(16.51)$ & 1.03 & 1.19 \\
\hline SPSQ & 31.41 (7.79) & $18.52(8.51)$ & 17.83 (10.79) & 1.58 & 1.44 \\
\hline LSAS-SR & $74.14(22.81)$ & $44.41(21.35)$ & $42.66(23.28)$ & 1.35 & 1.37 \\
\hline \multicolumn{6}{|l|}{ Bib $(n=29)$} \\
\hline SPS & $36.28(15.21)$ & $21.65(10.87)$ & 22.00 (10.33) & 1.11 & 1.10 \\
\hline SIAS & $45.14(17.41)$ & 33.83 (17.57) & 33.59 (17.90) & 0.65 & 0.65 \\
\hline SPSQ & 30.93 (9.32) & 17.55 (12.68) & $18.48(11.65)$ & 1.20 & 1.18 \\
\hline LSAS-SR & $62.90(26.81)$ & $42.55(30.26)$ & 43.38 (29.89) & 0.71 & 0.69 \\
\hline \multicolumn{6}{|l|}{$\operatorname{BibDG}(n=28)$} \\
\hline SPS & $40.68(16.53)$ & 24.39 (13.58) & $21.82(12.10)$ & 1.08 & 1.30 \\
\hline SIAS & $51.64(14.26)$ & $36.54(14.15)$ & $35.96(15.46)$ & 1.06 & 1.05 \\
\hline SPSQ & $33.43(8.96)$ & 18.68 (9.19) & 18.64 (9.74) & 1.63 & 1.58 \\
\hline LSAS-SR & 75.75 (22.08) & 43.89 (22.83) & $43.00(22.54)$ & 1.42 & 1.47 \\
\hline \multicolumn{6}{|l|}{ IAR $(n=29)$} \\
\hline SPS & 43.72 (18.61) & 28.17 (16.51) & $26.41(14.78)$ & 0.88 & 1.03 \\
\hline SIAS & $52.45(15.41)$ & $39.90(15.36)$ & $37.90(16.88)$ & 0.82 & 0.90 \\
\hline SPSQ & 33.83 (9.76) & $23.24(11.45)$ & 23.55 (10.73) & 1.00 & 1.00 \\
\hline LSAS-SR & $78.93(25.36)$ & $53.03(26.97)$ & 50.38 (22.89) & 0.99 & 1.18 \\
\hline
\end{tabular}


on the SIAS $(F=3.75$, d.f. $=1,135, P=0.055)$. The adjusted means of these measures indicated better long-term improvement in the

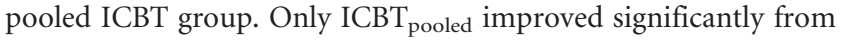
post-treatment test to 1-year follow-up, and on primary measures only $(t=2.52-4.02$, d.f. $=68, P=0.014-0.0001)$. The $\mathrm{ICBT}_{\text {pooled }}$ and $\mathrm{Bib}_{\text {pooled }}$ groups could not be differentiated with regard to change in secondary measures at either post-treatment or follow-up $\quad(F=0.04-0.63, \quad$ d.f. $=1,135, \quad P=0.98-0.43)$. Mean within-group effect sizes across all measures at post-treatment/ follow-up were $0.88 / 1.03$ for $\mathrm{ICBT}_{\text {pooled }}$ and $0.68 / 0.75$ for $\mathrm{Bib}_{\text {pooled. }}$ Mean between-group effect sizes for $\mathrm{ICBT}_{\text {pooled }} v$. $\mathrm{Bib}_{\text {pooled }}$ were 0.09 at post-treatment and 0.16 at follow-up.

Figure 2 shows weekly changes in LSAS-SR scores in the pooled ICBT and Bib groups in relation to the other conditions. Repeated-measurement ANOVA yielded a significant effect of time $(F=90.67$, d.f. $=11,1496, P<0.0001)$ and a tendency for a time $\times$ group $\left(\mathrm{ICBT}_{\text {pooled }} / \mathrm{Bib}_{\text {pooled }}\right)$ linear trend interaction $(F=3.15$, d.f. $=1,136, P=0.078)$. Further Bonferroni-corrected pairwise comparisons showed that the ICBT, Bib and BibDG groups were all significantly differentiated from WLC after the sixth week $(P<0.05)$, whereas a tendency $(P=0.062)$ was observed for IAR ( $v$. WLC) after the ninth week.

\section{Clinically significant improvement}

The number of participants meeting the criteria for clinically significant improvement is given in online Table DS2. The majority of participants (59-83\%) had improved on at least one social anxiety measure from baseline to post-treatment (the WLC group excepted) and from baseline to follow-up. In the WLC group $62 \%$ were improved after delayed treatment. The proportion of individuals who did not improve on any measure from baseline to follow-up was $23 \%$ in the $\mathrm{ICBT}_{\text {pooled }}$ group and $30 \%$ in the $\mathrm{Bib}_{\text {pooled }}$ group. The corresponding figures in

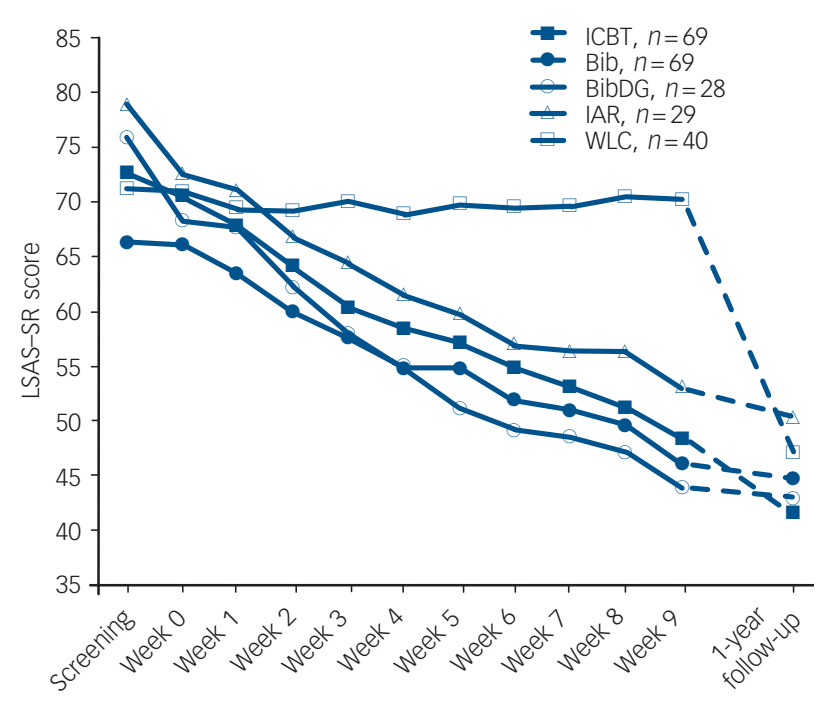

Fig. 2 Time course of improvement on the Liebowitz Social Anxiety Scale, self-report version (LSAS-SR). Data were recorded at screening, immediately before treatment (week 0 ) and then every week until post-treatment assessments after week 9 One-year follow-up data are also shown. Pooled data are shown for groups treated with pure bibliotherapy (Bib) and the internetdelivered cognitive-behavioural treatment package (ICBT). The waiting-list control group (WLC) was treated after the initial 9-week period. BibDG, bibliotherapy with access to an online discussion group; IAR, internet-delivered applied relaxation. the BibDG and IAR groups were $29 \%$ and $41 \%$. The proportion of clinically improved/unimproved participants did not differ across the active treatment arms at the post-treatment test $\left(\chi^{2}=2.98\right.$, d.f. $\left.=3, P=0.40\right)$ or at follow-up $\left(\chi^{2}=4.12\right.$, d.f. $=4$, $P=0.39)$.

\section{Treatment completion}

The number of completed modules is shown in online Table DS3. On average, participants completed 6.62 modules (s.d. $=2.62$ ) during the intended 9-week treatment period (trial 1: ICBT 7.35, Bib not assessed; trial 2: ICBT 6.41, Bib 5.86, BibDG 6.43, IAR 6.76). The mean number of completed modules did not differ significantly across groups $(F=1.51$, d.f. $=4,150, P=0.20)$. There were modest but mostly significant positive correlations between number of completed modules and change scores on social anxiety measures post-treatment $(r=0.15-0.29, P=0.07-0.002$, $n=154)$ and at follow-up $(r=0.23-0.29, \quad P=0.004-0.002$, $n=154)$.

\section{Treatment credibility}

Credibility ratings, obtained after the first treatment week, did not differ significantly between the treatments $(F=0.86$, d.f. $=3,189$, $P=0.46)$. Mean values were as follows: $\mathrm{ICBT}_{\text {pooled }}, 34.84$ (s.d.=7.84); $\quad$ Bib pooled, $33.74 \quad$ (s.d.=7.69); $\quad$ BibDG, 35.30 (s.d. =7.82); and IAR, 36.35 (s.d.=7.45).

\section{Discussion}

The magnitude and time course of improvements resulting from therapist-guided and unguided self-help programmes for social anxiety disorder were tested in two independent randomised trials, using an intention-to-treat model for data analyses.

\section{Bibliotherapy with and without discussion group}

Intriguingly, pure bibliotherapy had significant and reliable effects in people with social anxiety disorder. The magnitude of improvement did not differ significantly between the Bib and ICBT groups immediately after the 9 -week treatment programme even when pooled data (i.e. more highly powered analyses) were used, and comparable proportions met the criteria for clinically significant improvement. However, the highest within-group effect sizes were noted in the ICBT group. Moreover, only the ICBT group showed evidence of further improvement from post-treatment to follow-up, and a significant difference in favour of ICBT was noted on the LSAS-SR and SPS a year after treatment. Gains of pure bibliotherapy were, however, well maintained.

Although pure self-help has been shown to be efficacious in other conditions including anxiety disorders, ${ }^{13}$ to our knowledge only two prior randomised controlled trials (RCTs) have examined the efficacy of such self-help for social anxiety disorder. ${ }^{11,12}$ In contrast to our study, only small effects of pure bibliotherapy were found and this form of treatment was inferior to therapist-guided CBT. For example, effect sizes for change from baseline to post-treatment on the SPS and SIAS measures ranged between 0.28 and 0.38 respectively in participants treated with pure bibliotherapy, ${ }^{11,12}$ which can be compared with 0.83 and 0.65 for the Bib pooled group in our study. As the pre-treatment values on the SPS and SIAS measures were similar in all these studies, it is unlikely that initial differences in symptom severity can account for differential outcomes. A difference in the number of chapters read is also an unlikely explanatory factor because rather modest completion rates were noted in all three studies. 
One possibility is that the weekly assessments of LSAS-SR could have served as a motivational enhancer for bibliotherapy participants in our study. However, these assessments did not have a positive effect in the WLC group. The dissimilar effects of pure bibliotherapy can probably be attributed to variations in the structure and content of the written self-help programme. Although optimisation of text-based treatment programmes is an important topic for future research, our findings suggest that neither the pure self-help format, nor the inclusion of individuals with severe phobia, precludes significant treatment gains with bibliotherapy.

It could be argued that 'real world' bibliotherapy is inferior to bibliotherapy within the context of an RCT. For example, individuals purchasing a self-help book without any contact with a research team might be less motivated to complete the written treatment programme compared with those who participate in a clinical trial. Unfortunately it is not possible to evaluate real world bibliotherapy properly because scientific control cannot be achieved without assessments and at least some investigator contact. $^{12}$

Although time, assessments and contact with the study personnel were controlled for by the waiting-list condition, the motivational factor was not. Keeping the reader motivated is a major challenge for bibliotherapy and an online discussion group may be one way to enhance motivation and treatment adherence. Even though the statistical evaluation of our study did not demonstrate significant additional gains in the bibliotherapy arm with access to online group discussions, effect sizes were higher relative to pure bibliotherapy and, in fact, as high as in those treated with ICBT. The number of completed modules also tended to be higher in this arm relative to pure bibliotherapy. However, as noted in several other self-help studies, $6,9,10$ completion rates were not strongly associated with outcome, i.e. even limited exposure to the self-help programme had beneficial effects.

\section{Internet-delivered treatment with therapist guidance}

The effect sizes for ICBT compare well with those reported in our previous trials. ${ }^{4-6}$ Thus, short-term and long-term efficacy of internet-delivered CBT for social anxiety disorder has now been demonstrated in five RCTs in Sweden. The efficacy of online treatment for social anxiety disorder is also supported by three recent trials performed in Australia. ${ }^{9-11}$ However, studies that have evaluated text-based treatments not using the internet as a mean of delivery have also reported significant gains in people with social anxiety disorder. ${ }^{12,31}$ The current data further suggest that neither the internet format nor therapist feedback are necessary for obtaining clinical improvement because directly comparable gains were noted in the BibDG arm lacking these components. Interestingly, our previous trials did not demonstrate better effects of ICBT combined with two to five group therapy sessions, ${ }^{4,6}$ which raises questions as to the value of additional therapist-led contact, at least in the group context. On the other hand, we found a strong association between therapist input and effect size in another trial, ${ }^{32}$ also seen in a meta-analysis. ${ }^{2}$ Although more studies comparing self-help with face-to-face therapy clearly are needed, Rapee et al reported comparable effects of augmented self-help and standard group CBT for social anxiety disorder. ${ }^{12}$

\section{Specificity of the text-based treatment}

Treatment specificity for the text-based self-help manual for social anxiety disorder ${ }^{24}$ can be questioned, because participants treated with applied relaxation - which was based on a completely different manual - also improved to levels that were statistically indistinguishable from the other treatment arms. At the time the study was planned, meta-analytical evidence suggested that applied relaxation had only moderate effects, similar to pill placebo. ${ }^{8}$ Recent studies, however, have demonstrated that applied relaxation may be effective for at least some people with social anxiety disorder. ${ }^{33}$ Thus, a variety of treatment techniques may produce significant gains in this clinical population. It should also be noted that there was some overlap between applied relaxation and the standard self-help programme, for example with regard to psychoeducation and exposure exercises. Dismantling studies are needed to determine the active components in the different selfhelp programmes tested here. Comparisons with a credible placebo control are also necessary before it can be safely concluded that the cognitive-behavioural components are responsible for the therapeutic effects, although meta-analyses report lower effect sizes for placebo. ${ }^{8}$

\section{Time course of improvement}

To our knowledge, the time course of improvement has not previously been evaluated in studies of text-based self-help programmes. This type of data can be relevant for clinical planning and for handling low-intensity treatments. It might be expected that the effects of CBT, being learning-based, evolve rather slowly and progress more rapidly during the second half of the treatment period. For example, in a clinical study of social anxiety disorder, Heimberg et al noted that CBT was associated with fewer responders in comparison to phenelzine midway through treatment but that the response rate was approximately similar after the full 12 -week treatment period. ${ }^{34}$ However, in our study both the ICBT and Bib groups improved gradually and steadily. Intriguingly, more than half of the change at the post-treatment assessment had already been achieved after the first 4 weeks. Comparable response curves were noted in the other treatment arms, with the possible exception of the IAR group. Considerable gains may thus be expected early in the self-help treatment period, shedding light on the somewhat puzzling observation that substantial improvement occurs even though completion rates are moderate or low.

\section{Limitations}

Some limitations of our study require comment. First, because of moderate sample sizes (especially in trial 2), absence of differential improvement should be interpreted with power issues in mind. Unfortunately, we could only use the more highly powered (pooled) analyses when comparing the ICBT and Bib groups. Second, excluding people who were suicidal or had severe depression might limit the generalisability of our findings. These individuals are, however, usually considered too risky to include in research trials. Third, differential diagnosis could be an issue because, as in our previous trials, ${ }^{5,6}$ the telephone screening procedure did not permit in-depth psychiatric assessment. Comorbidity analyses could thus not be performed. Fourth, assessors were not masked with regard to the treatment assignment. However, all assessments were conducted online with standardised written instructions and automatic scoring, reducing the risk of reactivity or experimenter effects. Finally, we could not control for other treatments received during the 1-year follow-up period, which may affect interpretation of the long-term effects.

\section{Future directions and implications}

Future studies could compare pure bibliotherapy with therapistled group or individual CBT and examine if the bibliotherapy 
format could be further improved, for example by computerinteractive methods. This can be crucial as unguided programmes often lead to high withdrawal rates, at least on open-access websites. ${ }^{35}$ Studies could also evaluate whether bibliotherapy improves the effect of serotonin reuptake inhibitors or other anxiolytic agents. Finally, bibliotherapy might be evaluated in a stepped-care model in which more resource-demanding interventions are gradually offered to those who do not respond.

In conclusion, this study replicates the positive results of internet-delivered CBT previously demonstrated for individuals with social anxiety disorder, and also demonstrates that bibliotherapy without therapist input can lead to substantial long-lasting improvement, especially when supported with an online discussion group. This may have important clinical implications because bibliotherapy, being cheap and widely available, can be used as an augmentation to pharmacotherapy, or as the initial intervention before other therapies are offered.

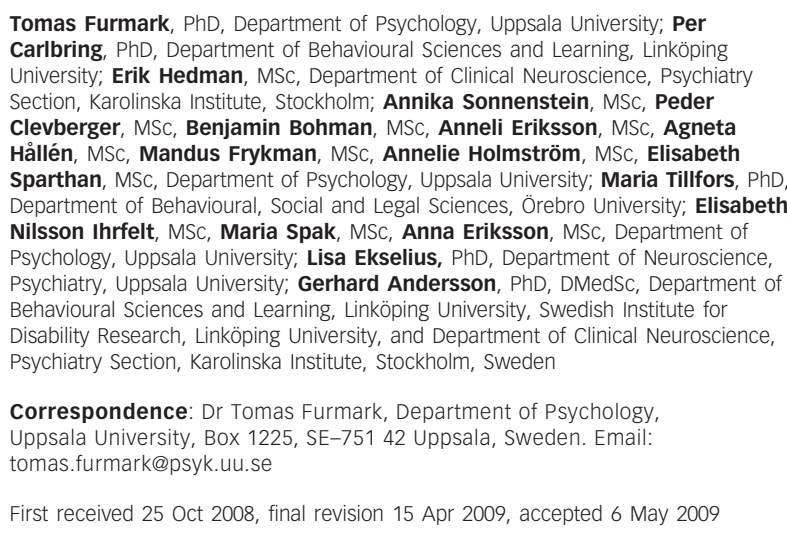

\section{Acknowledgements}

This study was supported by the Swedish Research Council.

\section{References}

1 Andersson G, Bergström J, Buhrman M, Carlbring P, Holländare F, Kaldo V, et al. Development of a new approach to guided self-help via the Internet. The Swedish experience. J Technol Hum Serv 2008; 26: 161-81.

2 Spek V, Cuijpers P, Nyklicek I, Riper H, Keyzer J, Pop V. Internet-based cognitive behaviour therapy for symptoms of depression and anxiety: a meta-analysis. Psychol Med 2007; 37: 319-28.

3 Carlbring P, Furmark T, Steczkó J, Ekselius L, Andersson G. An open study of internet-based bibliotherapy with minimal therapist contact via email for social phobia. Clin Psychol 2006; 10: 30-8.

4 Andersson G, Carlbring P, Holmström A, Sparthan E, Furmark T, Nilsson-Ihrfelt $E$, et al. Internet-based self-help with therapist feedback and in-vivo group exposure for social phobia: a randomized controlled trial. J Consult Clin Psychol 2006; 74: 677-86.

5 Carlbring P, Gunnarsdóttir M, Hedensjö L, Andersson G, Ekselius L, Furmark $T$. Treatment of social phobia: randomised trial of internet-delivered cognitive-behavioural therapy with telephone support. Br J Psychiatry 2007; 190: $123-8$

6 Tillfors $M$, Carlbring $P$, Furmark $T$, Lewenhaupt S, Spak M, Eriksson A, et al. Treating university students with social phobia and public speaking fears: internet delivered self-help with or without live group exposure sessions. Depress Anxiety 2008; 25: 708-17.

7 Acarturk C, Cuijpers P, van Straten A, de Graaf R. Psychological treatment of social anxiety disorder: a meta-analysis. Psychol Med 2009; 39: 241-54.

8 Fedoroff IC, Taylor S. Psychological and pharmacological treatments of social phobia: a meta-analysis. J Clin Psychopharmacol 2001; 21: 311-24.

9 Titov N, Andrews G, Schwencke G, Drobny J, Einstein D. Shyness 1: distance treatment of social phobia over the Internet. Aust N Z J Psychiatry 2008; 42 585-94.
10 Titov N, Andrews G, Schwencke G. Shyness 2: treating social phobia online: replication and extension. Aust N Z J Psychiatry 2008; 42: 595-605.

11 Titov N, Andrews G, Choi I, Schwencke G, Mahoney A. Shyness 3: randomized controlled trial of guided versus unguided internet-based CBT for social phobia. Aust N Z J Psychiatry 2008; 42: 1030-40.

12 Rapee RM, Abbott MJ, Baillie AJ, Gaston JE. Treatment of social phobia through pure self-help and therapist-augmented self-help. Br J Psychiatry 2007; 191: 246-52.

13 Cuijpers $P$, Schuurmans J. Self-help interventions for anxiety disorders: an overview. Curr Psychiatry Rep 2007; 9: 284-90.

14 Furmark T, Tillfors M, Everz P, Marteinsdottir I, Gefvert O, Fredrikson M. Social phobia in the general population: prevalence and sociodemographic profile. Soc Psychiatry Psychiatr Epidemiol 1999; 34: 416-24.

15 Svanborg P, Åsberg M. A new self-rating scale for depression and anxiety states based on the Comprehensive Psychopathological Rating Scale. Acta Psychiatr Scand 1994; 89: 21-8.

16 American Psychiatric Association. Diagnostic and Statistical Manual of Mental Disorders (4th edn, text revision) (DSM-IV-TR). APA, 2000.

17 First MB, Gibbon M, Spitzer RL, Williams JBW. Structured Clinical Interview for DSM-IV Axis I Disorders (SCID-I). American Psychiatric Press, 1997.

18 Baker SL, Heinrichs N, Kim HJ, Hofmann SG. The Liebowitz Social Anxiety Scale as a self-report instrument: a preliminary psychometric analysis. Behav Res Ther 2002; 40: 701-15.

19 Mattick RP, Clarke JC. Development and validation of measures of social phobia scrutiny fear and social interaction anxiety. Behav Res Ther 1998; 36: $455-70$.

20 Beck AT, Epstein N, Brown G, Steer R. An inventory for measuring clinical anxiety. Psychometric properties. J Consult Clin Psychol 1988; 56: 893-7.

21 Frisch MB, Cornell J, Villanueva M, Retzlaff PJ. Clinical validation of the Quality of Life Inventory: a measure of life satisfaction for use in treatment planning and outcome assessment. Psychol Assessment 1992; 4: 92-101.

22 Carlbring P, Brunt S, Bohman S, Austin D, Richards JC, Öst LG, et al. Internet vs. paper and pencil administration of questionnaires commonly used in panic/agoraphobia research. Comput Hum Behav 2007; 23: 1421-34.

23 Borkovec TD, Nau SD. Credibility of analogue therapy rationales. J Behav Ther Exp Psychiatry 1972; 3: 257-60.

24 Furmark T, Holmström A, Sparthan E, Carlbring P, Andersson G. Social FobiEffektiv Hjälp med Kognitiv Beteendeterapi [Social Phobia - Effective Help with CBT]. Liber, 2006.

25 Öst LG. Applied relaxation: description of a coping technique and review of controlled studies. Behav Res Ther 1987; 25: 379-409.

26 Carlbring $P$, Ekselius L, Andersson G. Treatment of panic disorder via the internet: a randomized trial of CBT vs. applied relaxation. $J$ Behav Ther Exp Psychiatry 2003; 34: 129-40.

27 Cohen J. Statistical Power Analysis for the Behavioral Sciences (2nd edn). Erlbaum, 1988.

28 Jacobson NS, Truax P. Clinical significance: a statistical approach to defining meaningful change in psychotherapy research. J Consult Clin Psychol 1991; 59: 12-9.

29 Furmark T, Tillfors M, Stattin H, Ekselius L, Fredrikson M. Social phobia subtypes in the general population revealed by cluster analysis. Psychol Med 2000; 30: 1335-44.

30 Fresco DM, Coles ME, Heimberg RG, Liebowitz MR, Hami S, Stein MB, et al. The Liebowitz Social Anxiety Scale: a comparison of the psychometric properties of self-report and clinician-administered formats. Psychol Med 2001; 31: 1025-35.

31 Abramowitz JS, Moore EL, Braddock AE, Harrington DL. Self-help cognitive-behavioral therapy with minimal therapist contact for social phobia: a controlled trial. J Behav Ther Exp Psychiatry 2009; 40: 98-105.

32 Palmqvist B, Carlbring P, Andersson G. Internet-delivered treatments with or without therapist input: does the therapist factor have implications for efficacy and cost? Expert Rev Pharmacoecon Outcomes Res 2007; 7: 291-7.

33 Clark DM, Ehlers A, Hackmann A, McManus F, Fennell M, Grey N, et al. Cognitive therapy versus exposure and applied relaxation in social phobia: a randomized controlled trial. J Consult Clin Psychol 2006; 74: 568-78.

34 Heimberg RG, Liebowitz MR, Hope DA, Schneier FR, Holt CS, Welkowitz LA et al. Cognitive behavioral group therapy vs phenelzine therapy for social phobia: 12-week outcome. Arch Gen Psychiatry 1998; 55: 1133-41.

35 Christensen H, Griffiths K, Groves C, Korten A. Free range users and one hit wonders: community users of an internet-based cognitive behaviour therapy program. Aust N Z J Psychiatry 2006; 40: 59-62. 